\title{
ENERGY LIFECYCLE MANAGEMENT. A NEW CONCEPT FOR MANAGING THE ENERGY EFFICIENCY IN MANUFACTURING PROCESSES
}

\author{
BATISTA PONCE, M[oises]; RONDAN, S[erafin]; SEBASTIAN, M[iguel] A[ngel]; GOMEZ, A[lvaro]; \\ MAYUET, P[edro] F[rancisco] \& MARCOS, M[ariano]
}

\begin{abstract}
Manufacturing floors possess a considerable number of particularities, which do that they are slightly repetitive and that the learning obtained in a floor are not literally exportable to others without a previous process of adjustment. This is especially relevant from the energetic point of view. Thus, energetic management of a manufacturing process must take into consideration a lot of aspect that can influence in the total energy consumption of the process. In this way, energetic savings can be strongly conditioned by the energy efficiency as a consequence of a good energy management. This paper contains the proposal of a new and specific methodology for the energetic management. This methodology has been so-called Energy Lifecycle Management (ELM) and it can be easily integrated in the management of the manufacturing process.

Key words: Manufacturing Process, Energetic Efficiency, Energetic Management, Energy Lifecycle Management, ELM
\end{abstract}

\section{INTRODUCTION}

Currently the performance of a manufacturing process must take into account economic, energetic and environmental aspects $[1,2]$. In particular, energetic performance influences directly in the productivity and in the competitiveness of the company, and it is not independent of economic and/or environmental performance [2-5]. Energetic management of a manufacturing floor has an interdependent character, which makes necessary a methodological systematizing in spite of its own specificities. Nevertheless, it must be sufficiently flexible in its applications in order to adapt specifically the solution to each one of the manufacturing floors in every moment [3,4].

Notwithstanding, there can be found barriers -mainly organizational and cultural- that make difficult the system implementation of the energy efficiency management. In this paper a specific methodology -that takes into account these barriers- for the energetic management has been designed. This methodology has been so-named Energy Lifecycle Management (ELM) and it can be easily integrated in the management of the manufacturing process.

\section{ELM: ENERGY LIFECYCLE MANAGEMENT. WHAT'S NEW?}

Energy Lifecycle Management (ELM) can be defined as a set of energetic analysis and management tools for the implementation of energetic management systems in the manufacturing floors $[2,5]$. These management tools can be grouped in four different blocks, Figure 1, according to [6-10]:

- Process lifecycle based energetic management, showing process evolution along its lifecycle.

- Data analysis based management, using viable and verifiable indexes.

- Process lifecycle coordinated management, through the coordination by an energetic manager along the useful life of the manufacturing floor.
- Management of the energetic culture and the energy policy, at all the levels of the company.

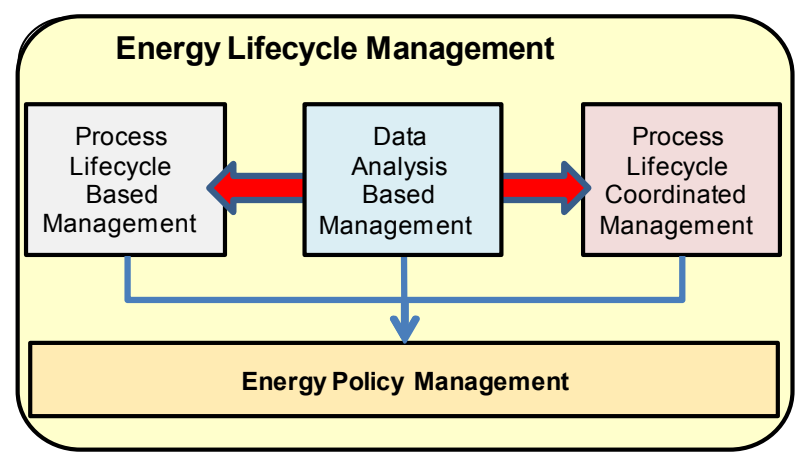

Fig. 1.Fundamental BLOCKS of Energy Lifecycle Management proposal

\section{ELM VIEWPOINTS: PRODUCT/PROCESS}

The main potential of energetic saving rests on the production style, which is demonstrated when the lifecycle is taken into account [2,5]. Thus, a previous analysis of the lifecycle must be carried out before proposing and managing improvements for increasing the energetic efficiency.

There are two ways: study based on process or study based on product. The energy efficiency of the process takes into account the efficiency of the manufacturing process including factors as the efficiency of the transport and distribution, Figure 2. As a consequent proposal, methods and operations of manufacture will be optimized, slightly efficient equipments will be replaced, logistic centers will be constructed, etc. Notwithstanding, it does not consider previous and posterior features. In this viewpoint, the energy efficiency of the suppliers, as well as the later product energetic consumption are factors with a marginal weight.

On the other hand, the analysis based on the energetic lifecycle of the product takes into account its global efficiency from the extraction and transformation of the raw materials up to its final use, including, so, the manufacturing process. In this sense, the influence of localized improvements on the useful life can be analyzed without losing data about the localized reductions or increases of the energetic consumption, including, so, the contribution to efficiency of the energetic suppliers and of the final use of the consumers.

A very interesting case study of the energetic lifecycle of the product can be found in [11] where results on its application to the manufacture of high stress steels for the automotive industry are reported [11]. In effect, these steels need higher energy consumption in their manufacturing process, but it is compensated by the savings in fuel oil as a result of a lower weight of the car [11]. 


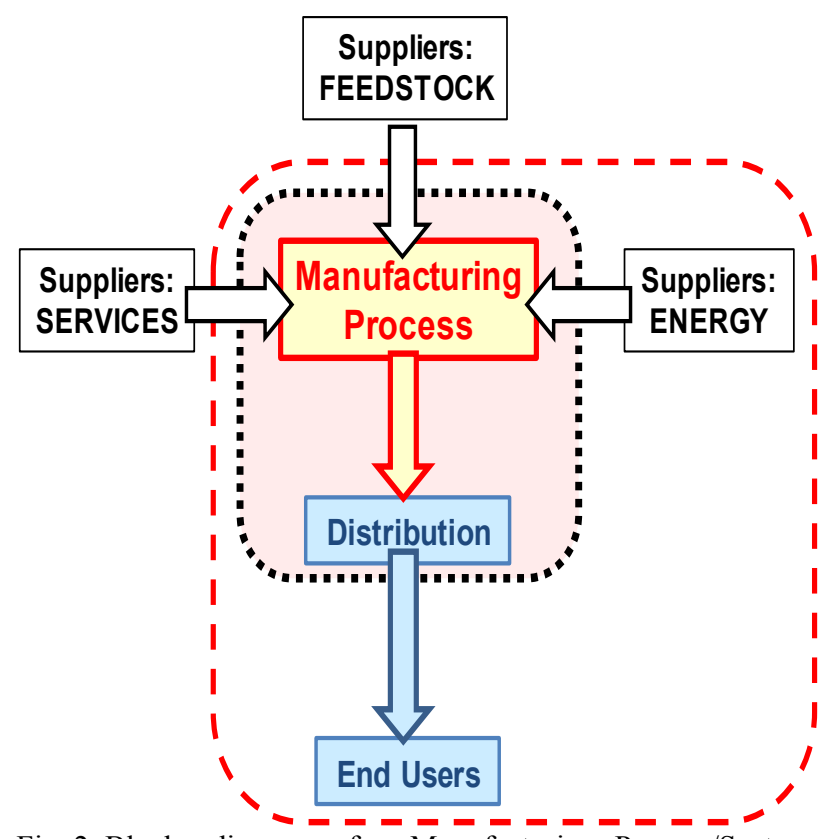

Fig. 2. Blocks diagram of a Manufacturing Process/System, including boundaries and, in a separated form, the different suppliers.

All that aforementioned can also be applied to other manufacturing systems, such as those related with Naval Industry or Power Transformers (low-medium voltage), as it can be observed in Figure 3. As a consequence of all that, it can be said that energy savings during the product useful lifecycle can be much higher than that reached in its manufacturing process.

As it can be deduced from the behavior paragraphs, from the process energetic lifecycle viewpoint the use of materials that need a major quantity of energy in its manufacturing process cannot be considered.

\section{CONCLUSIONS}

The Energy Lifecycle Management (ELM) methodology is a set of tools that make possible the energetic management of the manufacturing plants, as well as its efficiency. Product lifecycle energetic management might show important energetic savings related to the use and functionality of the products. An approach centered on the energetic management based on the product lifecycle exceeds the field of responsibility of the manufacturing floor and it does not give it a higher competitiveness in relation to its competence. Therefore, the energy efficiency must be contemplated from the point of view of the process lifecycle, and not from the product lifecycle by, mainly by two reasons:

- Energy management is a tool for obtaining a higher productivity and competitiveness. The product energy efficiency can influence on the added value and, therefore, in the perception of the product for the market. Nevertheless, once decided the product, there must be continuously searched the best production way; that will contribute competitiveness across the energy efficiency of the processes.

- Product lifecycle plays a relevant role at the moment of establishing policies, incentives, regulations, etc.

In summary, it must be reported that product based ELM can present very much major opportunities of efficiency that process based ELM. However, a great part of this potential of saving is out of the limits of the plant.

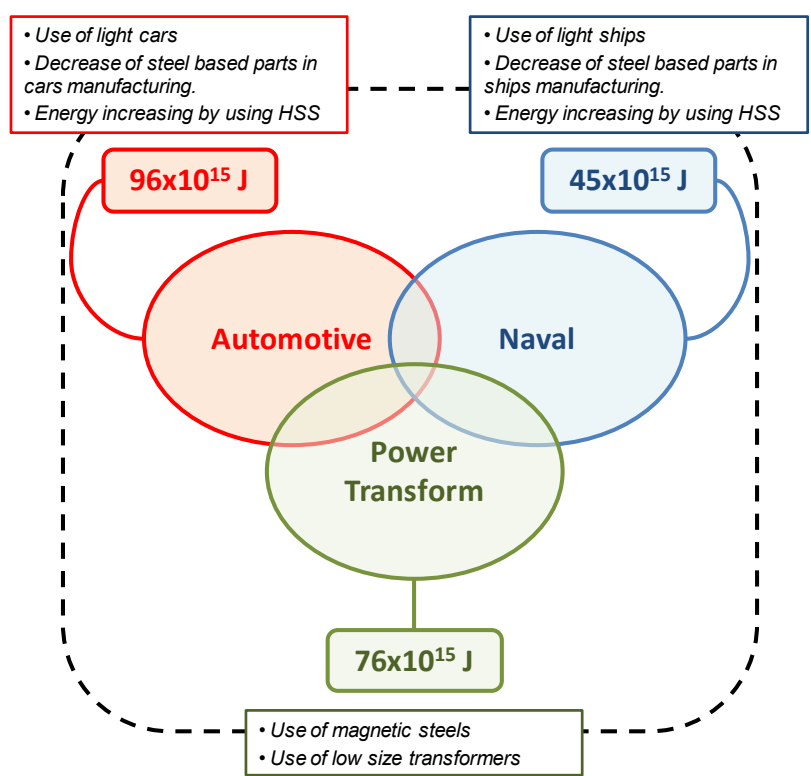

Fig. 3.An example of Energy Savings adapted from data recorded in [11].

\section{ACKNOWLEDGEMENTS}

This work has received financial support from the Spanish Government project DPI2008-06771-C04-01, from the Andalusian Government, and from the UE (FEDER).

\section{REFERENCES}

M. Alvarez, J. Salguero, J.A. Sanchez, M. Huerta and M. Marcos, SEM and EDS Characterisation of Layering TiOx Growth onto the Cutting Tool Surface in Hard Drilling Processes of Ti-Al-V Alloys, Advances in Materials Science and Engineering, Volume 2011, Article ID 414868, doi: $10.1155 / 2011 / 414868$

S. Rondan, M.A. Sebastian, M. Marcos, A methodology for the Energetic Efficiency Management in the Manufacturing Industry (in Spanish), Proc. II JORPRESI, Cadiz (Spain), 2010

K. Kounetas, K. Tsekouras, Are the Energy Efficiency Technologies efficient?, Economic Modeling (2009)

$\mathrm{K}$. Kounetas, K. Tsekouras, The energy efficiency paradox revisited through a partial observability approach, Energy Economics 30(2008) 2517-2536

S. Rondan, R. Bienvenido, M.A. Sebastian, A.Gómez, M. Marcos, Energetic Efficiency in the Industry. Key parameters identification for the electrical optimization, Proc. 3rd CIIEM, Portalegre (Portugal), 2009

Y. Okada, Competition and productivity in Japanese manufacturing industries, J. Japanese Int. Economies (2005) 586-616

International Energy Agency. Assessing Measures Of Energy Efficiency Performance And Their Application In Industry, 2008.

International Energy Agency. Worldwide Trends in Energy Use and Efficiency, 2008.

International Energy Agency. Progress with Implementing Energy Energy Efficiency Policies in the G8, 2009.

International Energy Agency. Innovations in National Energy Efficiency Strategies and Action Plans, Working Papers, 2009

International Energy Agency. Energy Use in the New Millenium, 2007 